\title{
N93-16880
}

\section{PREDICTION OF THERMAL CYCLING INDUCED MATRIX CRACKING}

\author{
by \\ Hugh L. McManus \\ Boeing Assistant Professor of Aeronautics and Astronautics \\ Massachusetts Institute of Technology \\ Cambridge, MA 02139
}

\begin{abstract}
Thermal fatigue has been observed to cause matrix cracking in laminated composite materials. A method is presented to predict transverse matrix cracks in composite laminates subjected to cyclic thermal load. Shear lag stress approximations and a simple energy-based fracture criteria are used to predict crack densities as a function of temperature. Prediction of crack densities as a function of thermal cycling is accomplished by assuming that fatigue degrades the material's inherent resistance to cracking. The method is implemented as a computer program. A simple experiment provides dita on progressive cracking of a laminate with decreasing temperature. Existing data on thermal fatigue is also used. Correlations of the analytical predictions to the data are very good. A parametric study using the analytical method is presented which provides insight into material behavior under cyclical thermal loads.

\section{Background}

Unprotected structure in space goes through wide, cyclical swings in temperature. These cycles are severe $\left(+/-150^{\circ} \mathrm{F}\right.$ in LEO, $+/-250^{\circ} \mathrm{F}$ in GEO) and a long-life structure such as the Space Station must withstand up to 175,000 cycles in a lifetime. Advance composite materials are widely and successfully used for space structure, but transverse cracks in the plies of composite structures have been observed to form under thermal cycling loads. In some critical applications, this cracking may degrade performance, and even threaten the integrity of the space structure. Greater understanding of this phenomena is necessary to predict, avoid, and/or live with transverse cracking in composite materials.
\end{abstract}

\section{Approach}

Given appropriate material properties, the layup and geometry of a composite laminate, and a thermal loading history, we will predict the resulting distribution of matrix cracks, and the resulting degraded laminate properties. An analytical model is implemented as a computer program to provide the predictions. It is verified by correlation with experiments, some performed as part of this effort and some garnered from the literature.

Analytical Model Thermal or mechanical loading stores energy in the laminate. Some of this energy is released by the formation of a transverse crack. Typically, these cracks appear suddenly across the full height and width of a ply, parallel to the fibers. The amount of energy released can be approximated by a shear lag solution of the stresses in the vicinity of the crack (Figure 1) and a simple energy method-if the energy released $\Delta G$ is greater than the energy necessary to form the crack $G_{c}$, the crack will form (see, for example, [1]). Multiple cracks are handled by predicting a crack densily $\rho$, which is the inverse of the average crack spacing (Figure 2). Predictions based on a single parameter $\rho$ assume an unrealistically even crack spacing, but can be used to calculate either the maximum possible crack density, or the minimum density. To provide an idea of the true crack density, a direct Monti-Carlo simulation of crack accumulation is performed, assuming cracks initiate at random locations in the material. Fatigue is accounted for by assuming that cyclic loading decreases the material toughness factor $G_{c}[2]$. 
Computer Program The method was implemented as a FORTRAN computer code that runs on Macintosh or other computers. It requires layup geometry and material properties as inputs, an will solve for crack density and degraded material properties at a given temperature, or provide plotable tables of crack density and material properties as functions of either decreasing temperature, or number of thermal fatigue cycles at a constant minimum temperature.

Experiments A simple experiment was performed to measure crack density as a function of decreasing temperature. P75-934 laminates with a layup of [0/ $/ 902] \mathrm{s}$ were cooled to progressively lower temperatures in an environmental chamber, and matrix cracks counted under an optical microscope. Available thermal fatigue data on a variety of materials and layups was also used $[3,4]$.

Correlations The measured crack density as a function of temperature followed the lower bound predicted by the analysis (Figure 3). It was determined, by comparing the measured crack distributions to predicted distributions, that this was due to (unmodeled) macroscopic irregularities in the strength of the plies. Use of the minimum crack density prediction, as well as the values of some geometric and material parameters that were not available, were set by this correlation. Then the code was used in predictive mode (with no adjustment of parameters) to correlate fatigue data. A typical result is shown in Figure 4. The correlations are excellent.

Parametric Studies The code was used to study the effects of material properties, ply thicknesses, and layups on the cracking of laminates under both progressively dropping temperatures and thermal cycling. It was found that colder temperature and/or fatigue caused increasing cracking, with no true "saturation" level ever being reached (although variations in crack densities did become very small under some circumstances). Increasing material toughness $G_{c}$ delayed the appearance of the first crack and lowered crack densities. Using thinner plies delayed the appearance of the first crack, but resulted in more cracking later. Cracking in thin plies also had much less effect on laminate properties than cracking in thick plies. The layup had a complex, non-intuitive effect on the cracking behavior of the laminate.

\section{Conclusions and Recommendations}

The technique developed here looks very promising, both as a design and analysis tool, and as a way to increase understanding of the phenomena of transverse crack formation. More data is needed, notably $\mathrm{G}_{c}$ of materials as functions of both temperature and number of thermal cycles. More direct correlation testing would be interesting, particularly comparing the results of mechanical vs. thermal loads. More analytical work is needed in the areas of damage initiation modeling (i.c. how do cracks form?), crack distribution modeling (where do they go?), and links to delamination and laminate failure. Success would lead to an accurate analytical tool for the design of reliable composite space structure.

\section{References}

1. Laws, N., and Dvorak, G. J., "Progressive Transverse Cracking in Composite Laminates", Journal of Composite Materials, Vol. 22, 1988, pp. 900-916.

2. Petitpas, E., Renault, M., and Valentin, D., "Fatigue Behavior of Cross-Ply CRFP Laminates made of T300 or T400 Fibres", International Journal of Fatigue, Vol. 12, 1990, pp. 245-251.

3. Bowles, D. E., and Shen, J., "Thermal Cycling Effects on the Dimensional Stability of P75 and P75-T300(Fabric) Hybrid Graphile/Epoxy Laminates", presented at the 33rd International SAMPE Symposium and Exhibition, Anahiem CA, March 1988.

4. Unpublished data communicated by Steve Tompkins, LaRC. 

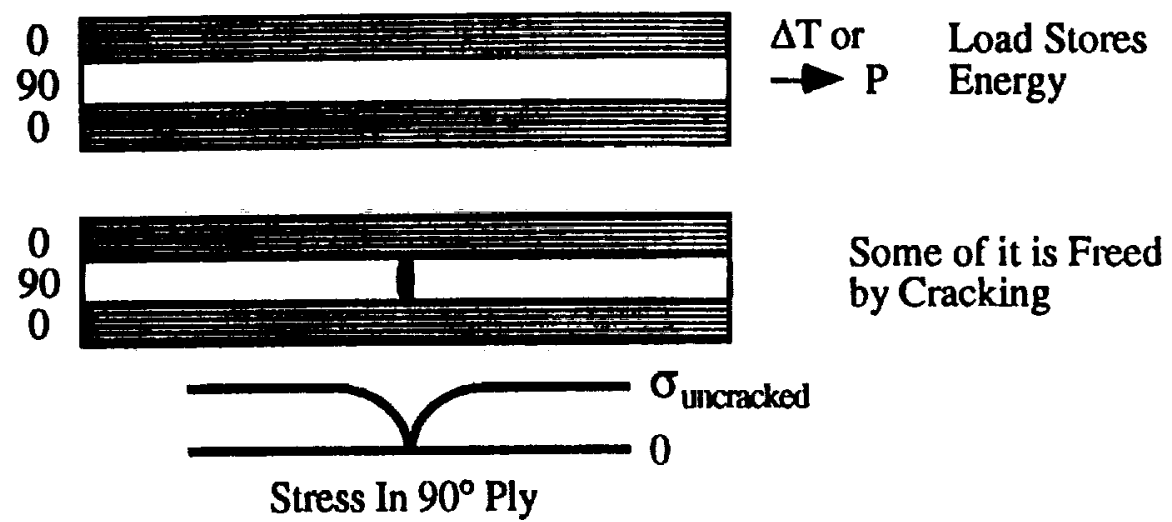

Figure 1. Energy stored in a laminate is freed by the appearance of a crack. Shown is an edgeon view of a laminate. The crack is assumed to extend through the $90^{\circ}$ ply to the other edge.

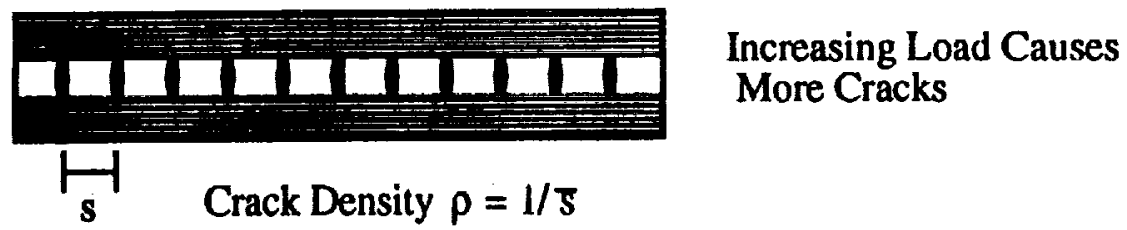

Figure 2. Progressive cracking measured by crack density $\rho$.

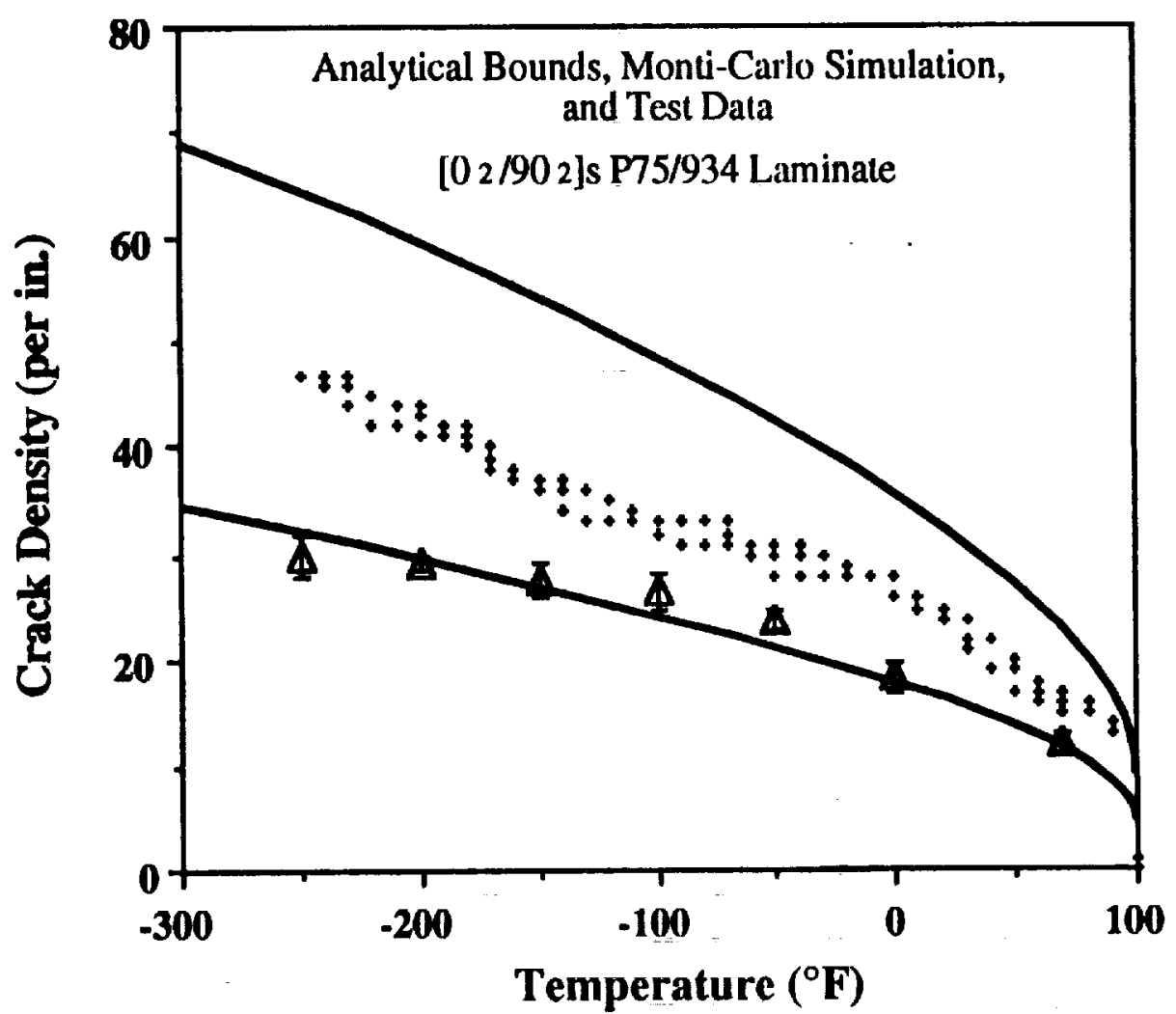

Figure 3. Predicted upper and lower bounds of crack density (solid lines), Monti-Carlo model predictions (dots) and test data (triangles, with bars showing range of three specimens). 


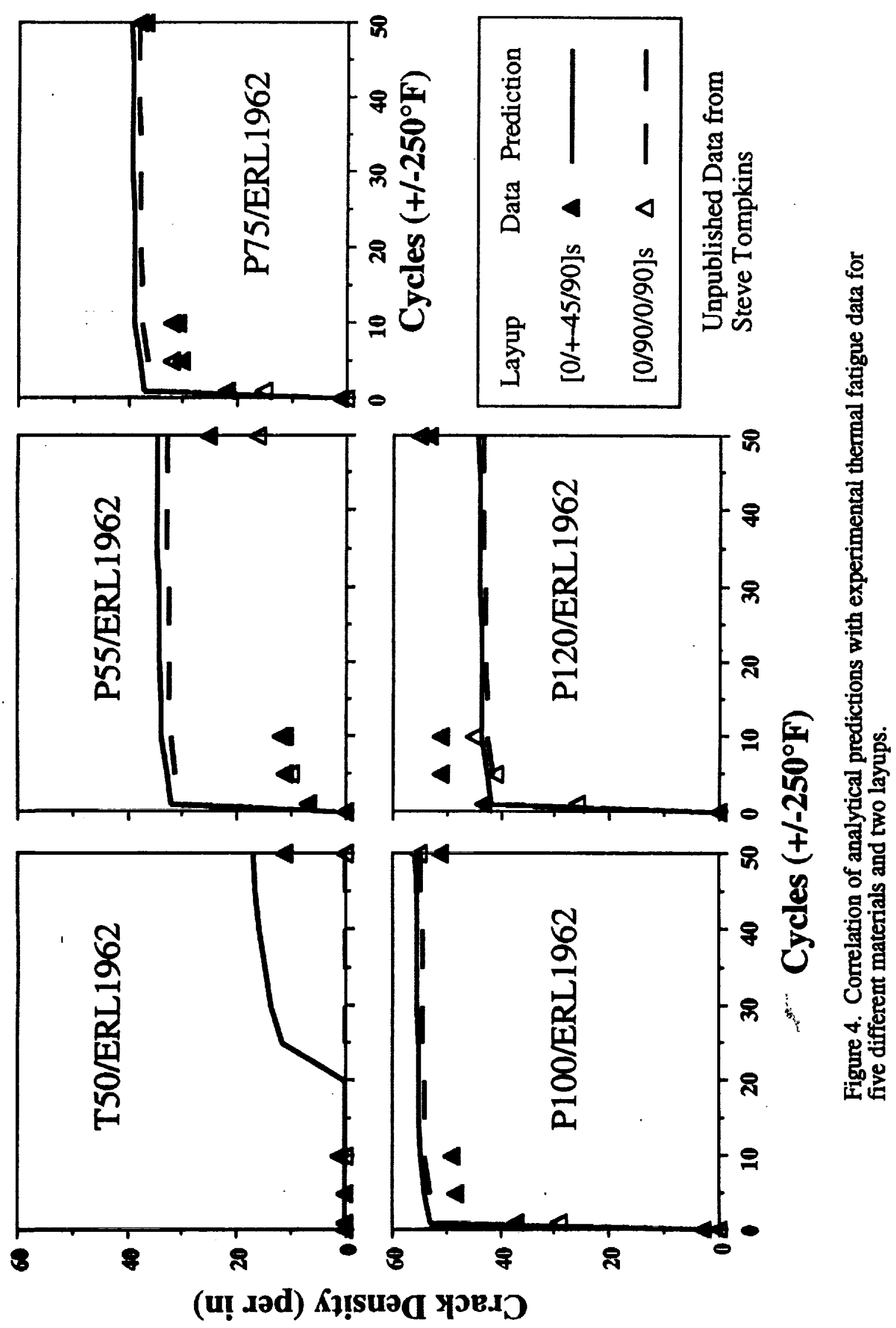

